\title{
Magnetic Eddy-Current, Fluid and Thermal Coupled Model for the Finite Element Behavior of Gas-Insulated Bus Bars
}

\author{
Xiaowen $\mathrm{Wu}^{1, \mathrm{a}}$, Nianguang $\mathrm{Zhou}^{1}$ and Chunming $\mathrm{Pei}^{2}$ \\ ${ }^{1}$ State Grid Hunan Electric Power Corporation Research Institute, Changsha 410007, China \\ ${ }^{2}$ China Electric Power Research Institute, Wuhan 430074, China \\ awxwwhu@163.com
}

\begin{abstract}
Keywords: Finite element method, eddy current, thermal analysis, fluid dynamics, sensitivity analysis, product development.

Abstract. This paper describes a coupled magnetic eddy-current, fluid and thermal finite-element model to analyze the steady and transient thermal behavior of a three-phase gas-insulated bus bar (GIB). The magnetic eddy-current field is indirectly coupled into the fluid and thermal fields through the power loss density while considering the temperature dependent electrical conductivity. As a new methodology, multiple species transport technique is introduced into the thermal model using computational fluid dynamics (CFD), circumventing the difficulty of applying temperature and geometry dependent heat transfer coefficient on the model boundaries. Good agreement is found between the CFD and experimental results. The proposed methodology is further used to predict the steady and transient thermal behaviors of the GIB under the effects of various gas pressures, gas types, electrical loads, and ambient temperatures, which provides useful sensitivity information for GIB designers in the product development process.
\end{abstract}

\section{Introduction}

Knowledge of the thermal behavior plays an important role in understanding the improvement of the design and manufacture processes of gas-insulated bus bars (GIBs) in order to keep the operation temperature at safety levels. The analysis of temperature rise process in GIB is a coupled magnetic eddy current, fluid and thermal problem. In currently available thermal models, the finite-element method (FEM) has been most widely used [1-5]. However, the heat transfer problem is less understood for the difficulty in applying the convective heat transfer coefficient on the enclosure surface, which depends on many factors such as surface location, model geometry, as well as ambient temperatures [5]. Moreover, the sensitivity analysis, which provides useful information identifying the important parameters affecting the steady and transient temperature rise of the GIB, is never mentioned in the models. In addition to conduction and radiation, a significant portion of the heat generated in the conducting components of the GIB gets dissipated into the surroundings by convection of the insulation gas and ambient air. Thus, the convective heat transfer coefficient is a paramount parameter in the thermal analysis of GIB.

In this paper, the surrounding air is introduced into the model, and the multiple species transport technique is proposed to calculate the material properties of the gas mixture of the ambient air and the insulation gas. The convection heat transfer inside and outside the GIB is calculated by computational fluid dynamics (CFD). The methodology is further used to analyze the thermal behaviors of the GIB affected by the parameters of gas pressure, gas type, electrical loading, and ambient temperature.

\section{Finite Element Model}

Eddy Current Field Model. The considered three-phase GIB is composed of aluminum alloy enclosure, the insulation gas and three aluminum conductors, as shown in Fig. 1. The electromagnetic field diffusion is governed by the Maxwell's equations while the displacement current is neglected. The reluctivities of the conductor and enclosure are constant, and the GIB is assumed to be long enough. Thus, the problem becomes 2-D in the $x-y$ plane. In the analysis, electromagnetic field is considered up 
to infinity and then vanishes at the boundary $\Gamma_{1}$. The 2-D eddy current diffusion problem in a time-harmonic regime can be stated as follows [6]:

$$
\left\{\begin{array}{l}
\Omega: v \nabla^{2} A+j \omega \sigma(T)\left(-A+\frac{J_{\mathrm{s}}}{j \omega \sigma(T)}\right)=0 \\
\Gamma_{1}: A=0
\end{array}\right.
$$

where $A$ is the magnetic vector potential, $\sigma(T)$ is the electric conductivity dependent on temperature $T$, $v$ is the reluctivity, $J_{\mathrm{s}}$ is the source current density, $\Omega$ is the solution region, $\Gamma_{1}$ is the Dirichlet boundary condition.

The total current density $J$ is decomposed into source and eddy current densities, the total current $I$ for the conductor of the cross-section area $S_{\mathrm{c}}$ can be expressed as [6]

$$
\iint_{S_{\mathrm{c}}} j \omega \sigma(T)\left(-A+\frac{J_{\mathrm{s}}}{j \omega \sigma(T)}\right) d s=\iint_{S_{\mathrm{c}}} J d s=I
$$

Applying the Galerkin method to discrete (1) and (2), the power loss $Q$ in the conductor per unit length can be calculated by [7]

$$
Q=\frac{1}{2} \sum_{i=1}^{n} \frac{9 \% 0 \%}{\sigma_{i}(T)} S_{i}
$$

where $s_{i}$ is the element area, $n$ is the total element number of the conductor, $g_{i} /$ is the complex total current density for element $i, 9 \%$ is the complex conjugate of $9 / c$.

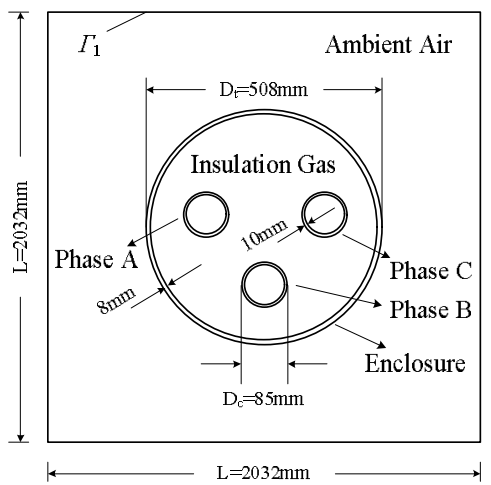

Fig. 1. Solution region of the three-phase gas insulated bus bars.

Thermal Model. The power losses generated in the GIB are dissipated into the surroundings mainly by convection and radiation. It has been reported that the heat convection approximately reaches up to $40 \%$ of all heat transferred from the GIB [8]. Therefore, the accurate calculation of convective heat transfer is paramount in the thermal analysis process. The determination of the temperature dependent convective heat transfer coefficient by the nondimensional parameters that include the Grashof (Gr), Prandtl (Pr), Rayleigh ( $\mathrm{Ra}$ ), and Nusselt (Nu) numbers is a complicated process, especially when the surface shape is irregular. However, with the multiple species transport technique, the challenge could be circumvented.

Introducing the ambient air into the solution region of the thermal model, the convective heat transfer is calculated by CFD analysis. In the thermal model, the ambient air and insulation gas are fluids with different properties. The flow of these fluids is treated as multiple species transport problem. A single momentum equation is solved for the flow field.

In GIB, the bulk properties of the gas mixture of the ambient air and insulation gas can be calculated as a linear combination of the properties of these species [9].

$$
\alpha=\sum_{i=1}^{2} \alpha_{i} Y_{i}
$$




$$
\sum_{i=1}^{2} Y_{i}=1
$$

where $\alpha$ is the density, thermal conductivity, dynamic viscosity, or specific heat of the gas mixture, $\alpha_{i}$ and $Y_{i}$ are the material property and mass fraction of the insulation gas or ambient air corresponding to $\alpha$, respectively.

In the thermal analysis process, the specific heat of the gas mixture is considered constant. The density $\rho$ variation with the temperature and gas pressure is evaluated by the ideal gas law

$$
\rho=\rho_{0} \frac{p T_{0}}{p_{0} T}
$$

where $p$ is the gas pressure, $\rho_{0}$ is the density at $0^{\circ} \mathrm{C}, T_{0}$ and $p_{0}$ are the reference temperature and pressure, respectively.

The thermal conductivity $\lambda$ and dynamic viscosity $\mu$ are nearly not influenced by the gas pressure. Their variation with temperature is obtained by the Sutherland's law, which are, respectively, expressed as [10]

$$
\begin{gathered}
\lambda=\lambda_{0}\left(\frac{T}{T_{0}}\right)^{1.5} \frac{T_{0}+S}{T+S} \\
\mu=\mu_{0}\left(\frac{T}{T_{0}}\right)^{1.5} \frac{T_{0}+S}{T+S}
\end{gathered}
$$

where $\lambda_{0}$ and $\mu_{0}$ are, respectively, the thermal conductivity and dynamic viscosity of the gas mixture at $0 \stackrel{\circ}{ } \mathrm{C}, S$ is the Sutherland temperature.

According to the theory of CFD, the natural convection inside and outside the GIB satisfying the following governing equations [7]:

1) Continuity Equation:

$$
\frac{\partial \rho}{\partial t}+\frac{\partial(\rho u)}{\partial x}+\frac{\partial(\rho v)}{\partial y}=0
$$

2) Momentum Equation:

$$
\begin{aligned}
& \frac{\partial(\rho u)}{\partial t}+\frac{\partial(\rho u u)}{\partial x}+\frac{\partial(\rho v u)}{\partial y}=-\frac{\partial p}{\partial x}+\mu \nabla^{2} u+S_{x} \\
& \frac{\partial(\rho v)}{\partial t}+\frac{\partial(\rho u v)}{\partial x}+\frac{\partial(\rho v v)}{\partial y}=\rho g-\frac{\partial p}{\partial y}+\mu \nabla^{2} v+S_{y}
\end{aligned}
$$

3) Energy Equation:

$$
\frac{\partial}{\partial t}(\rho C T)+\frac{\partial}{\partial x}(\rho u C T)+\frac{\partial}{\partial y}(\rho v C T)=\lambda \nabla^{2} T+Q_{v}
$$

where $u$ and $v$ are the fluid velocity in $x$ and $y$ direction and are zero for solid domains, respectively, $S_{x}$ and $S_{y}$ are the source terms, $g$ is the gravitational acceleration, $C$ is the specific heat, $Q_{\mathrm{v}}$ is the heat source.

As shown in Fig. 1, the heat transfer of the GIB is an open boundary problem, higher accuracy results as outer boundary expands, which in turn increase the size of the problem. Generally with FEM analysis, the dimension of the solution region is chosen 4 times the diameter of the GIB. At the outermost boundary of the solution region, the temperature is assumed to be constant.

Radiation heat transfer occurs between the conductors and enclosure and between the enclosure and ambient air. Two types of radiation boundary conditions are used in the model. One open-type enclosure surface radiation boundary condition on the outer tank surface is applied as follows. 


$$
-\lambda \frac{\partial T}{\partial n}=\varepsilon \sigma\left(T^{4}-T_{\mathrm{a}}^{4}\right)
$$

where $n$ is the normal direction of tank surface, $\varepsilon$ is emissivity, $\sigma$ is Stefan-Boltzmann constant.

The other one is the closed-type enclosure radiation boundary condition between the outer surface of the conductors and the inner surface of the enclosure, which is expressed as [10]

$$
-\lambda \frac{\partial T}{\partial n}=\varepsilon F_{i j} \sigma\left(T_{i}^{4}-T_{j}^{4}\right)
$$

where $F_{i j}$ is the view factor, $T_{i}$ and $T_{j}$ are the temperatures of the radiating two surfaces.

No-slip boundary conditions on $\Gamma_{1}$ are applied in the model stated as

$$
\left.u\right|_{\Gamma_{1}}=\left.v\right|_{\Gamma_{1}}=0
$$

\section{Results and Discussions}

The whole solution region is meshed with 37734 elements and 106661 nodes. The finite element calculations of eddy-current, fluid and thermal fields are run on a computer with Intel (R) Duad-Core processor with a speed of $3.20 \mathrm{GHz}$ and $4 \mathrm{~GB}$ of RAM.

Power Losses. As the power losses are temperature dependent, an iterative method between magnetic eddy current and thermal fields has been used. Initial temperatures are assumed in the conductors and enclosure. Then, the thermal field is analyzed, if the error between the calculated temperature and the initial value is larger than $5 \%$, the initial temperatures are updated. This process repeats until the convergence criterion is met.

The total current density distribution along the enclosure surface at different load currents is shown in Fig. 2. The induced eddy current at the position adjacent to the conductors is much higher than that of the other locations on the enclosure surface. As the symmetrical structure and load currents, the eddy current densities induced by phase $\mathrm{A}$ and $\mathrm{C}$ have the same distribution. Comparing with the induced eddy current density with phase $\mathrm{A}$ and $\mathrm{C}$, the phase-B induced eddy current density is smaller in amplitude, which is mainly attributed to the different spatial locations in the GIB. The power losses of the components in the GIB are given in Table 1, which are used as heat source input for the thermal analysis.

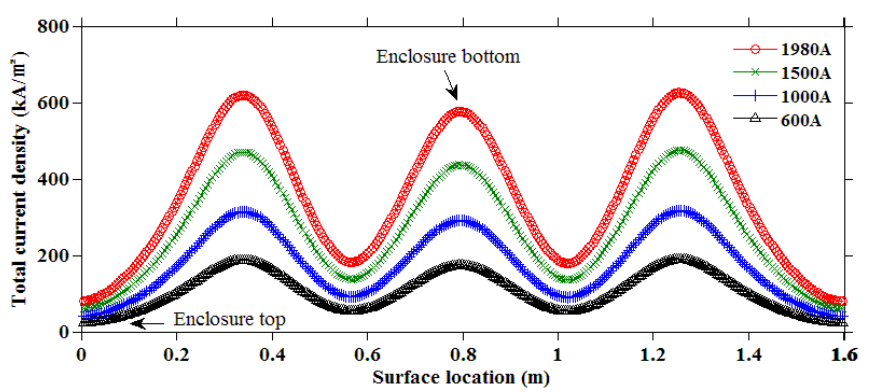

Fig. 2. Total current density distribution on the enclosure surface.

Table 1 Power losses of the components in the GIB

\begin{tabular}{ccccc}
\hline \hline $\begin{array}{c}\text { Load current } \\
(\mathrm{A})\end{array}$ & $\begin{array}{c}\text { Phase A } \\
(\mathrm{W} / \mathrm{m})\end{array}$ & $\begin{array}{c}\text { Phase B } \\
(\mathrm{W} / \mathrm{m})\end{array}$ & $\begin{array}{c}\text { Phase C } \\
(\mathrm{W} / \mathrm{m})\end{array}$ & $\begin{array}{c}\text { Enclosure } \\
(\mathrm{W} / \mathrm{m})\end{array}$ \\
\hline 600 & 5.21 & 5.25 & 5.20 & 3.02 \\
1000 & 14.59 & 14.60 & 14.54 & 8.26 \\
1500 & 34.60 & 34.80 & 34.49 & 19.03 \\
1980 & 63.54 & 63.46 & 63.32 & 34.32 \\
\hline \hline
\end{tabular}

Stead- and Transient-State Thermal Behaviors. With the CFD analysis, the temperature and fluid velocity distribution of the whole solution region can be obtained, as shown in Fig. 3. It can be observed that the temperature and fluid velocity are symmetrically distributed. When the ambient 


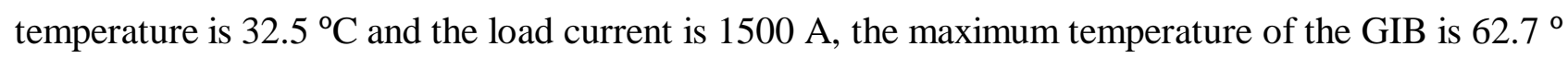
$\mathrm{C}$ located at the conductors of phase $\mathrm{A}$ and $\mathrm{C}$. Compared with the temperature of phase $\mathrm{A}$ and $\mathrm{C}$, that of phase B is several centigrade degrees lower. Generally, the three-phase conductors are assumed to be isothermal. Natural convection occurs in the solution region. Under the effect of buoyancy, the ambient air and insulation gas move upward. The maximum fluid velocity is about $0.2 \mathrm{~m} / \mathrm{s}$ at the interface of the ambient air and the enclosure.

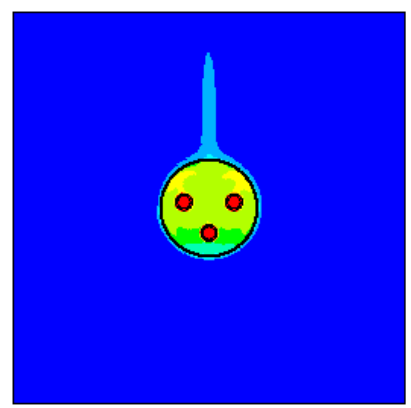

(a) Temperature distribution

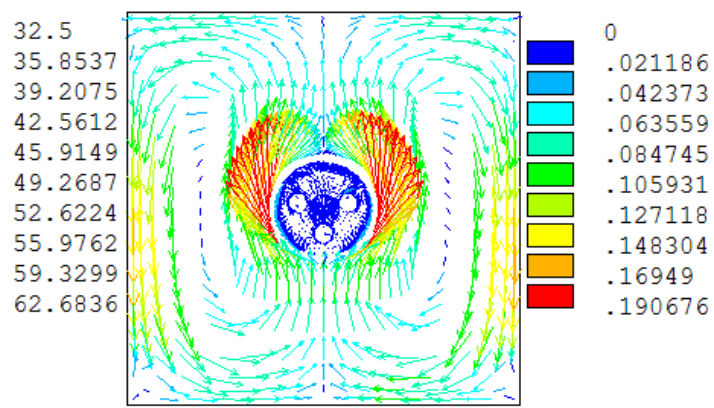

(b) Fluid velocity distribution

Fig. 3. Temperature and fluid velocity distribution of the whole solution region at $1500 \mathrm{~A}$.

According to Newton's law of cooling, the convective heat transfer coefficient distribution on the enclosure surface is shown in Fig. 4. The maximum value is about $3 \mathrm{~W} /\left(\mathrm{m}^{2} \dagger \mathrm{K}\right)$ located at the middle height of the enclosure. The minimum heat transfer coefficient is less than $1 \mathrm{~W} /\left(\mathrm{m}^{2} \dagger \mathrm{K}\right)$ at the enclosure top. Large discrepancy exists between the maximum value and the minimum one. The result is deemed to be the actual distribution, as the coefficient is found to be continuously changing along the enclosure surface and also to be temperature dependent.

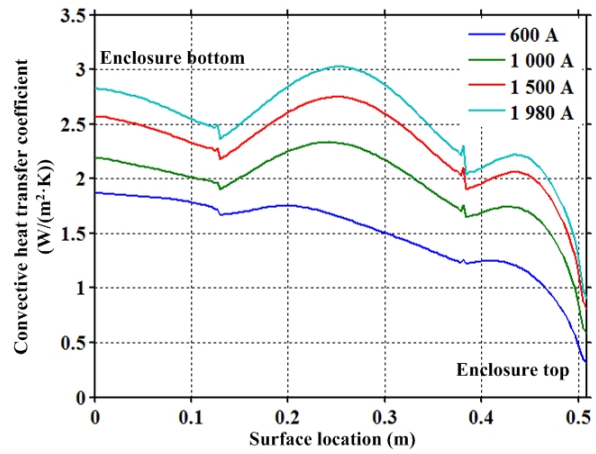

Fig. 4. Convective heat transfer coefficient on the enclosure surface.

Time variation of the GIB temperature with changing load currents in 12 hours is shown in Fig. 5. The GIB temperature changes rapidly with the variation of load currents. Compared with the enclosure, the conductor temperature is much more sensitive to the variation of load current. With transient thermal analysis, the time constant of the conductor and enclosure can be obtained, which is useful for the dynamic prediction of the temperature rise process in GIB.

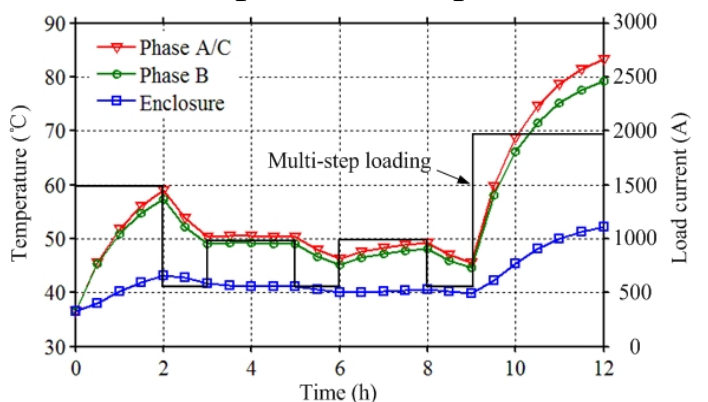

Fig. 5. Time variation of the GIB temperature with changing load current.

The proposed model is validated by experimental results obtained from a $126 \mathrm{kV}$ three-phase GIB prototype insulated with $\mathrm{N}_{2}$ gas of $0.4 \mathrm{MPa}$. Table 2 gives the comparison of the calculated and tested results. It is observed that the calculated and tested temperatures are in good agreement. 
Sensitivity Analysis. Several calculations are carried out to investigate the sensitivity of the GIB temperature to several selected parameters which are the load current, ambient temperature, gas pressure and insulation gas type.

Variation of the GIB temperature with load current and ambient temperature is given in Fig. 6. Nonlinear relationship is found between the GIB temperature and the load current. The temperature rises as the load current increases. At rated load current, the conductor temperature rise reaches over $50.7 \mathrm{~K}$ and that of the enclosure reaches $20 \mathrm{~K}$. It can be concluded that the GIB temperature is largely dependent on the load current. Moreover, it is also observed that the temperature rise of GIB is nearly not influenced by the variation of ambient temperature, which means if the relationship between the GIB temperature and the load current were obtained the GIB temperature in steady state can be predicted with the load current, regardless of the variation of ambient temperature.

Table 2 Comparison of the calculated and tested temperatures $\left({ }^{\circ} \mathrm{C}\right)$

\begin{tabular}{|c|c|c|c|c|c|c|}
\hline \multirow{2}{*}{$\begin{array}{l}\text { Load current } \\
\text { (A) }\end{array}$} & \multicolumn{2}{|c|}{ Phase A/C } & \multicolumn{2}{|c|}{ Phase B } & \multicolumn{2}{|c|}{ Enclosure } \\
\hline & FEM & tested & FEM & tested & FEM & tested \\
\hline 600 & 43.7 & 45.3 & 43.2 & 43.9 & 40.1 & 40.8 \\
\hline 1000 & 44.7 & 47.3 & 43.3 & 45.3 & 35.2 & 35.4 \\
\hline 1500 & 63.0 & 63.4 & 60.6 & 60.1 & 43.2 & 43.3 \\
\hline 1980 & 87.2 & 85.8 & 83.2 & 80.2 & 54.5 & 52.5 \\
\hline
\end{tabular}

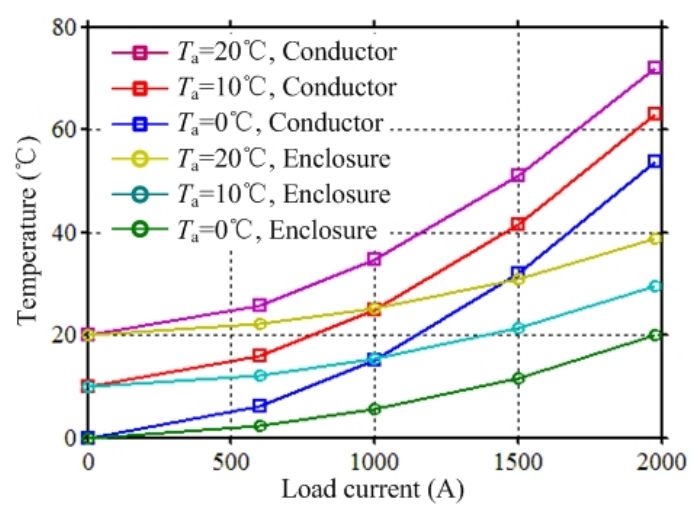

Fig. 6. GIB temperature variation with load current and ambient temperature.

Fig. 7 gives the temperature variation of the GIB with the pressure of the insulation gas when the ambient temperature is $32.5^{\circ} \mathrm{C}$ and the load current is $1500 \mathrm{~A}$. The GIB temperature seems to be not sensitive to the pressure variation of the insulation gas. As the gas pressure increases from 0.1 to 0.6 $\mathrm{MPa}$, the temperature of the conductor decrease from 64.8 to $61.5{ }^{\circ} \mathrm{C}$, and that of the enclosure decrease from 43.5 to $43.0^{\circ} \mathrm{C}$. Only $3.3^{\circ} \mathrm{C}$ difference of the conductor is found in a large range of gas pressure.

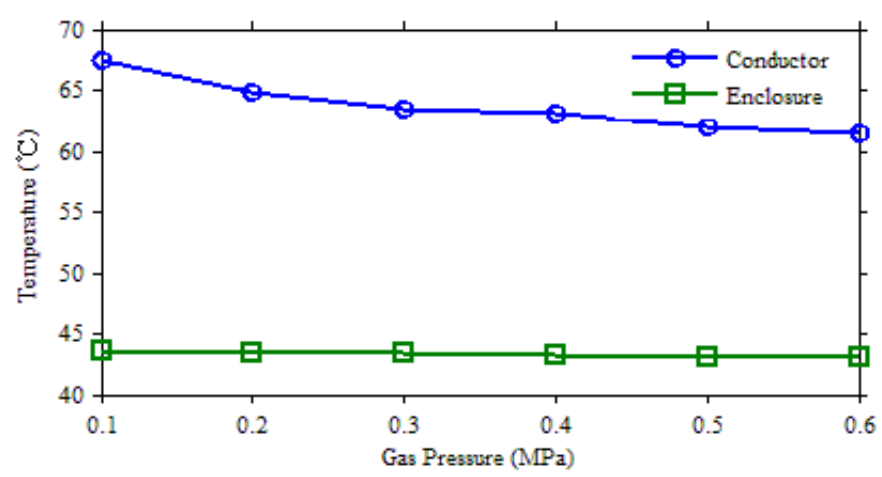

Fig. 7. Variation of the GIB temperature with gas pressure.

In GIB pressurized $\mathrm{SF}_{6}$ and $\mathrm{SF}_{6} / \mathrm{N}_{2}(20 / 80 \%)$ are usually used as the insulation gas. The GIB temperature variation with the insulation gas is investigated. The temperature distribution of the GIB is similar with that shown in Fig. 3(a). It is found that under rated load current the operation temperature 
of the GIB insulated with $\mathrm{SF}_{6}$ is about ${ }^{\circ} \mathrm{C}$ lower than that insulated with $\mathrm{SF}_{6} / \mathrm{N}_{2}$ (20/80\%). Compared with the parameter of gas pressure, the GIB temperature seems to be more sensitive to the type of insulation gas.

\section{Conclusions}

In this paper, a coupled magnetic eddy-current, fluid and thermal finite-element model is proposed to analyze the steady and transient thermal behavior of a three-phase GIB, in which the multiple species transport technique is employed and the complicated calculation of temperature and surface location dependent convective heat transfer coefficient is avoided. The effectiveness of the proposed model is validated with measurements. Sensitivity analysis is carried out to investigate the variation of the GIB temperature to the parameters of the load current, ambient temperature, gas pressure and insulation gas type.

\section{References}

[1] J.K. Kim, S.C. Hahn and K.Y. Park. IEEE Transactions on Magnetics Vol. 41 (2005), p. 1636-1639.

[2] S.L. Ho, Y. Li and X. Lin. IEEE Transactions on Magnetics Vol. 43 (2007), p. 1433-1436.

[3] R. Benato and F. Dughiero. IEEE Transactions on Magnetics Vol. 39 (2003), p. 1741-1744.

[4] H.K. Kim, J.K. Jung and K.Y. Park. IEEE Transactions on Magnetics Vol. 40 (2004), p. 1326-1329.

[5] S.W. Kim, H.H. Kim and S.C. Hahn. IEEE Transactions on Magnetics Vol. 38 (2002), p. 921-924.

[6] M. Rachek and S.N. Larbi. IEEE Transactions on Magnetics Vol. 44 (2008), p. 4739-4746.

[7] S. Pawar, K. Joshi and L. Andrews. IEEE Transactions on Power Delivery Vol. 27 (2012), p. 156-163.

[8] M.Z. Degefa, M. Lehtonen and R.J. Millar. IEEE Transactions on Power Delivery Vol. 27 (2012), p. 1662-1669.

[9] W.D. Bennon and F.P. Incropera. Int. J. Heat Mass Transfer Vol. 30 (1987), p. 2161-2170.

[10]M.B. Eteiba, M.M.A. Aziz and J.H. Shazly. IEEE Transactions on Power Delivery Vol. 23 (2008), p. 1457-1463. 